\title{
Economics of Residues Incorporation and Phosphorus Application for Direct Seeded Rice and Wheat under Saline Soil
}

\author{
Imdad Ali Mahmood ${ }^{*}$, Arshad Ali1, Muhammad Zahid Kiani' ${ }^{1}$, Armghan Shahzad ${ }^{2}$, \\ Tariq Sultan', Hussain Shah ${ }^{3}$, Muhammad Arshadullah1, Badar-uz-Zaman' ${ }^{1}$ \\ ${ }^{1}$ Land Resources Research Institute, Islamabad, Pakistan \\ ${ }^{2}$ National Institute for Genomics and Advanced Biotechnology, PARC-Institute of Advanced Studies in \\ Agriculture (The University of Agriculture, Peshawar), National Agricultural Research Centre, Islamabad, \\ Pakistan \\ ${ }^{3}$ Crop Disease Research Institute, Islamabad, Pakistan \\ Email: *imdad20260@yahoo.com
}

Received 30 July 2015; accepted 12 September 2015; published 15 September 2015

Copyright (C) 2015 by authors and Scientific Research Publishing Inc.

This work is licensed under the Creative Commons Attribution International License (CC BY). http://creativecommons.org/licenses/by/4.0/

cC) (7) Open Access

\section{Abstract}

Two-year long field study was conducted using a permanent layout to investigate the economics of crop residues incorporation ( $2 \mathrm{t}^{-h^{-1}} \mathrm{a}^{-1}$ and $\mathrm{P}$ application $\left(0,40,80\right.$ and $120 \mathrm{~kg} \mathrm{P}_{2} \mathrm{O}_{5} \mathrm{ha}^{-1}$ ) to directly sowing of rice and wheat crops gown under naturally salt-affected calcareous soil $\left(\mathrm{EC}_{\mathrm{e}}=4.59 \mathrm{dS}\right.$ $\mathrm{m}^{-1} ; \mathrm{pH}_{\mathrm{s}}=8.38 ; \mathrm{SAR}=6.57\left(\mathrm{mmol}_{\mathrm{c}} \mathrm{L}^{-1}\right)^{1 / 2} ; \mathrm{CaCO}_{3}=3.21 \%$; Extractable $\mathrm{P}=4.07 \mathrm{mg} \cdot \mathrm{kg}^{-1}$; sandy clay loam) at farmers field in district Hafizabad during the year 2012-13. Split plot design (crop residues in main plots and $P$ application in sub plots) was followed with three replications. Agronomic data on growth and yield were collected at the time of each crop maturity. Maximum growth and yield of both the crops were harvested from the plots where $\mathrm{P}_{2} \mathrm{O}_{5}$ was applied @ $80 \mathrm{~kg} \cdot \mathrm{ha}^{-1}$ along with crop residues incorporation. On an average of two years, maximum paddy $\left(3.26 \mathrm{t}^{\left.\cdot \mathrm{ha}^{-1}\right)}\right.$ and wheat grain ( $3.56 \mathrm{t} \cdot \mathrm{ha}^{-1}$ ) yield were produced with $\mathrm{P}$ application @ $80 \mathrm{~kg} \mathrm{P}_{2} \mathrm{O}_{5} \mathrm{ha}^{-1}$ along with crop residues incorporation. Although, the yield harvested with this treatment $\left(80 \mathrm{~kg} \mathrm{P}_{2} \mathrm{O}_{5} \mathrm{ha}^{-1}+\mathrm{crop}\right.$ residues) performed statistically equal to $120 \mathrm{~kg} \mathrm{P}_{2} \mathrm{O}_{5} \mathrm{ha}^{-1}$ without crop residues incorporation during both the years, however, on an average of two years, grain yield of directly sowing rice and subsequent wheat was significantly superior $(22 \%$ and $24 \%$ respectively) than that of higher $P$ rate $\left(120 \mathrm{~kg} \cdot \mathrm{ha}^{-1}\right)$ without crop residues. Overall, continuous two-year crop residues incorporation further increased (17\%) paddy yields during the follow up year of crop harvest. Economic analyses of both the crops were carried out to choose the best treatment with adequate economic benefits as compared to those without crop residue incorporation. Maximum net benefit of Rs =

${ }^{*}$ Corresponding author.

How to cite this paper: Mahmood, I.A., et al. (2015) Economics of Residues Incorporation and Phosphorus Application for Direct Seeded Rice and Wheat under Saline Soil. Agricultural Sciences, 6, 934-942.

http://dx.doi.org/10.4236/as.2015.69090 
108,680/- for direct seeded rice and $\mathrm{Rs}=99,362 /$ - for wheat grown with $80 \mathrm{~kg} \mathrm{P}_{2} \mathrm{O}_{5}$ ha-1 $^{-1}$ application under crop residues incorporation was determined. Among $P$ application treatments without crop residues incorporation, the maximum net benefit ( $R s=75,874 /$ - and $R s=65,725 /-$ ) and highest residual values $(49,809$ and 39,160$)$ for direct seeded rice and wheat respectively, were obtained with extended $P$ application rate $\left(120 \mathrm{~kg} \mathrm{P}_{2} \mathrm{O}_{5}\right.$ ha- $\left.^{-1}\right)$ which was not again as much as that of $80 \mathrm{~kg} \mathrm{P}_{2} \mathrm{O}_{5}$ ha-1 $^{-1}$ application with crop residues incorporation.

\section{Keywords}

\section{Crop Residues Incorporation, P Application, Direct Seeded Rice and Wheat, Saline Soil, Economic Analysis}

\section{Introduction}

With world population now more than seven billion is a timely moment for such an assessment to boost up the grain production for fulfilling the mounting demands. Population growth and economic burden are exerting the pressure on arable lands. Intensive cropping are exhausting nutrients from soil equilibrium gradually which is among the major constraints to get optimum yields. The specter of possible changes in traditional agriculture could be a step forward to release these pressures. It is the time to consider how we can move "towards sustainability" towards a vision of natural resources management that supports current population's demands otherwise leaving the future generations on an equitable soil degradation owing to gradual nutrient depletion. Soil is critical component of the resource base upon which a successful agriculture depends. To move towards sustainability, agriculture and natural resource management interests must recognize that they are equal partners in the effort. The challenges are to adapt and extend our knowledge about soil health to develop economically productive, culturally appropriate and environmentally sound systems. A flexible ongoing process is necessary to set research priorities to support inherently dynamic agricultural techniques.

In Pakistani, totals extents off salt-affected areas is 6.3 mha, of which 1.89 mha is saline, 1.89 mha is permeable saline-sodic, 1.02 mha is impermeable saline-sodic while sodic-soils is only 0.028 mha. Provinces wised distributions off saline's patches outs of $1.89 \mathrm{mha}, 0.94 \mathrm{mha}$ is in the Punjab, $0.5 \mathrm{mha}$ in Sindh and $0.45 \mathrm{mha}$ is in Khyber Pakhtun Khawa [1]. Soil salinity and P fixation reduce activity of soil microorganisms. Nutrient mining due to intensive cropping and practice of imbalance fertilizers applications is the main examples of soil resources degradation. A significant reduction in the yield of rice and wheat owing to these reasons is $68 \%$ and $64 \%$, respectively, causing a loss estimate from 0.3 to 1.0 billion dollar per annum [2]. The interrelated apprehensions of rising population definitely impose economic pressures; intensified land use and environmental degradation at local and regional levels are serious issues to concern. Combinations of biological and societal resources are required to make successful agricultural production managements and its sustainability will necessitate the changes in philosophy and operating procedures for improving productivity. A major limitation in the rice-wheat cropping system is the short time between rice harvesting and wheat cultivation and any delay in planting adversely affects crop yields. As the result of improved farm machinery convenience, a large area under rice and wheat crops are being harvested with combined harvester which leaves behind a massive loose straw whose removal or exploitation in a short time period is not so easy. Rice is grown on 2.58 mha with annual straw production of about 4 million tons [3]. The situation compels farmers to burn them for preparation their lands for timely sowing of subsequent crops [4] [5]. Circumstances after the harvest of wheat crop are also the same. Crop residues are rich source of plant nutrients that farmers demolish through burning which not only causes nutrient losses but also pollutes the environment. In addition to these restrictions, $\mathrm{P}$ fixation in our soils due to calcareousness and high $\mathrm{pH}$ are other constraints considerably reducing crop yields and under saline conditions, its availability is further declined. The growing crop plants under such environment demand relatively higher nutrition to reach the potential yields. Since the prices of $\mathrm{P}$ fertilizers are becoming out of the reach of resource poor farmers day by day as a result they don't be anxious about nourishing their growing crop plants with balanced fertilization [6]. The left over residues could be recycled if its burning is discouraged. Generally, a large portion of nutrients taken up by the plants remains in the straw which can be utilized for the growth of 
subsequent crops through their incorporation [7]. In many studies, recycling of crop residues is reported to increase the nutrient status of the soils and hence crop productivity [8]-[10].

Traditionally transplanting of rice is a very hard and difficult process of cultivation which requires expensive labour and extensive machinery tools for puddling as well. Consequently, directly sowing of rice is an option which saves all these expenses and complexities. Keeping all these points in view, two year field study using a permanent layout was conducted under naturally saline soil to investigate the economics of crop residue incorporation as well as $\mathrm{P}$ application and their impact on direct seeded rice paddy and wheat grain yields.

\section{Materials and Methods}

A two year study using a permanent layout was conducted under marginal saline soil of rice-wheat cropping system at farmers field in Wachhoki Kalan, Kankah Dogran-Hafizabad Road, district Hafizabad $\left(\mathrm{EC}_{\mathrm{e}}=4.59\right.$ $\mathrm{dS} \cdot \mathrm{m}^{-1} ; \mathrm{pH}_{\mathrm{s}}=8.38 ; \mathrm{SAR}=6.57\left(\mathrm{mmol}_{\mathrm{c}} \cdot \mathrm{L}^{-1}\right)^{1 / 2} ; \mathrm{CaCO}_{3}=3.21 \%$; Extractable $\mathrm{P}=4.07 \mathrm{mg} \cdot \mathrm{kg}^{-1} ;$ Sandy clay loam) during 2012-13. The experiment was laid out according to split plot design with three replications. Planting methods i.e., direct seeding with and without crop residue (wheat) incorporation @ $2 \mathrm{t} \cdot \mathrm{ha}^{-1}$ were kept in main plots and various $\mathrm{P}$ doses $\left(0,40,80\right.$ and $\left.120 \mathrm{~kg} \mathrm{P}_{2} \mathrm{O}_{5} \mathrm{ha}^{-1}\right)$ were applied in sub plots.

Recommended basal dose of $\mathrm{N} @ 100 \mathrm{~kg} \cdot \mathrm{ha}^{-1}$ (half at sowing time and remaining half at tillering stage) and $\mathrm{K} @ 50 \mathrm{~kg} \cdot \mathrm{ha}^{-1}$ as SOP were applied to all the plots at the time of sowing. Soaked seed (for $24 \mathrm{~h}$ ) of rice cv. Supper-2000@ $40 \mathrm{~kg} \cdot \mathrm{ha}^{-1}$ was broad-casted uniformly. The same inputs were applied to intermediate wheat crop. Effective weedicides were used to control weeds and the crop was grown to upto maturity. All agronomic requirements and plant protection measures were met throughout the growth period whenever required. At maturity, each crop was harvested and direct seeded rice paddy and wheat grain yields were recorded.

The economic analysis of crop residues incorporation and four $\mathrm{P}$ application rates to direct seeded rice and wheat crops was computed by using the method as described earlier [11].

\section{Results and Discussion}

\subsection{Growth and Yield of Direct Seeded Rice and Wheat Crops}

On an average of two years data, maximum paddy $\left(3.26 \mathrm{t}^{\mathrm{h}} \mathrm{h}^{-1}\right)$ and wheat grain $\left(3.56 \mathrm{t} \cdot \mathrm{ha} \mathrm{a}^{-1}\right)$ yields were produced with P application @ $80 \mathrm{~kg} \mathrm{P}_{2} \mathrm{O}_{5} \mathrm{ha}^{-1}$ alongwith crop residues incorporation (Table 1) which was comparable with higher $\mathrm{P}$ rate $\left(120 \mathrm{~kg} \cdot \mathrm{ha}^{-1}\right)$ under no crop residues incorporation. The paddy and wheat grain yields produced by this treatment showed $22 \%$ and $24 \%$, respectively additional yield over control $\left(0 \mathrm{~kg} \mathrm{P} \mathrm{ha}^{-1}+\right.$ crop residues). Under crop residues incorporation, further increase in $\mathrm{P}$ application $\left(120 \mathrm{~kg}_{2} \mathrm{O}_{5} \mathrm{ha}^{-1}\right)$ caused 6\% paddy yield reduction as compared to the $\mathrm{P}$ application @ $80 \mathrm{~kg} \mathrm{P}_{2} \mathrm{O}_{5} \mathrm{ha}^{-1}$. Crop residue incorporation positively contributed in grain yield of direct seeded rice and subsequent wheat particularly during second year. This was most probably due to complete decomposition and mineralization of added crop residues that enriched the soil with mineral nutrients in addition to improvement in soil physical condition by ameliorating toxic effects of

Table 1. Average direct seeded rice and wheat yields $\left(\mathrm{kg} \cdot \mathrm{ha}^{-1}\right)$ 2011-12.

\begin{tabular}{|c|c|c|c|c|}
\hline Direct Seeded Rice Yield & T1 & $\mathbf{T} 2$ & T3 & T4 \\
\hline +CR (Grain) & 2685 & 2870 & 3262 & 3080 \\
\hline +CR (Straw) & 6853 & 6953 & 7447 & 7170 \\
\hline -CR (Grain) & 1724 & 2171 & 2715 & 3062 \\
\hline -CR (Straw) & 4491 & 5627 & 6194 & 6356 \\
\hline \multicolumn{5}{|l|}{ Wheat Yield } \\
\hline +CR (Grain) & 2652 & 3194 & 3560 & 3259 \\
\hline +CR (Straw) & 5836 & 6285 & 6576 & 6358 \\
\hline -CR (Grain) & 1591 & 2054 & 2877 & 3320 \\
\hline -CR (Straw) & 3577 & 4433 & 5263 & 5832 \\
\hline
\end{tabular}

$\mathrm{T} 1=0 \mathrm{~kg} \mathrm{P}_{2} \mathrm{O}_{5} \mathrm{ha}^{-1} ; \mathrm{T} 2=40 \mathrm{~kg} \mathrm{P}_{2} \mathrm{O}_{5} \mathrm{ha}^{-1} ; \mathrm{T} 3=80 \mathrm{~kg} \mathrm{P}_{2} \mathrm{O}_{5} \mathrm{ha}^{-1} ; \mathrm{T} 4=120 \mathrm{~kg} \mathrm{P}_{2} \mathrm{O}_{5} \mathrm{ha}^{-1}$; +CR = With Crop Residue; -CR = Without Crop Residue. 
saline ions. Moreover, water and P retention capacity might have also been improved due to added crop residues that retained comparatively excess moisture and $\mathrm{P}$ availability for a longer time. Besides, production of acid farming substances by microbial activities and partial pressure of $\mathrm{CO}_{2}$ released during crop residues decomposition decreased soil $\mathrm{pH}$ and enhanced $\mathrm{P}$ availability and other necessary plant nutrients which encouraged healthy plant growth and hence yields. Similar points of view have also been documented by [12] [13]. Further, adequate $\mathrm{P}$ fertilization promoted vigorous plant growth that ultimately improved number of grains per panicle resulting in increased yields of direct seeded rice and succeeding wheat crop [14] [15]. The increase in yield due to crop residues incorporation as well as $\mathrm{P}$ application has also been well documented by [16]-[20].

\subsection{Partial/Budget Analysis of Direct Seeded Rice and Wheat Crops}

Partial budget analysis for $\mathrm{P}$ application rates (Table 2 and Table 3) showed that all $\mathrm{P}$ application rates under crop residues incorporation gave higher benefit than that of without crop residues incorporation. However maximum net benefit for direct seeded rice and wheat crops was calculated from P application @ $80 \mathrm{~kg} \mathrm{P}_{2} \mathrm{O}_{5}$ $\mathrm{ha}^{-1}$ with crop residues incorporation under saline soil. This treatment for direct seeded rice and wheat again performed to be superior than that of elevated $\mathrm{P}$ application rate $\left(120 \mathrm{~kg} \mathrm{P}_{2} \mathrm{O}_{5} \mathrm{ha}^{-1}\right)$ without crop residues incorporation. Whereas, minimum net benefit was obtained from the plots receiving $40 \mathrm{~kg} \mathrm{P}_{2} \mathrm{O}_{5}$ ha $^{-1}$ without crop residues incorporation. Correspondingly, P application @ $80 \mathrm{~kg} \mathrm{P}_{2} \mathrm{O}_{5} \mathrm{ha}^{-1}$ with crop residues incorporation also demonstrated the highest Cost Benefit Ratio (CBR) for direct seeded rice and wheat.

Table 2. Partial budget analysis for direct seeded rice grown with and without CR under saline soil.

\begin{tabular}{|c|c|c|c|c|}
\hline Average Yield $\left(\mathrm{kg}^{2} \cdot \mathrm{ha}^{-1}\right)$ 2012-13 & T1 & T2 & T3 & T4 \\
\hline \multicolumn{5}{|c|}{$+\mathbf{C R}$} \\
\hline Paddy & 2685 & 2870 & 3262 & 3080 \\
\hline Straw & 6853 & 6953 & 7447 & 7170 \\
\hline TCV & 26,625 & 27,105 & 27,585 & 28,065 \\
\hline $10 \%$ less Paddy Yield & 268.5 & 287.0 & 326.2 & 308.0 \\
\hline 10\% less Straw Yield & 685.3 & 695.3 & 744.7 & 717.0 \\
\hline Adjusted Grain Yield & 2417 & 2583 & 2936 & 2772 \\
\hline Adjusted Straw Yield & 3427 & 6258 & 6702 & 6453 \\
\hline Income (Grain) & 84,578 & 90,405 & 102,753 & 97,020 \\
\hline Income (Straw) & 17,133 & $31,288.5$ & $33,511.5$ & 32,265 \\
\hline Gross Income (Paddy + Straw) & 101,710 & 121,694 & 136,265 & 129,285 \\
\hline Net Benefit (Rs ha' ${ }^{-1}$ ) & 75,085 & 94,589 & 108,680 & 101,220 \\
\hline \multicolumn{5}{|c|}{-CR } \\
\hline Paddy & 1724 & 2171 & 2715 & 3062 \\
\hline Straw & 4491 & 5627 & 6194 & 6356 \\
\hline TVC & 24,625 & 25,105 & 25,585 & 26,065 \\
\hline $10 \%$ less Paddy Yield & 172.4 & 217.1 & 271.5 & 306.2 \\
\hline $10 \%$ less Straw Yield & 449.1 & 562.7 & 619.4 & 635.6 \\
\hline Adjusted Paddy Yield & 1552 & 1954 & 2444 & 2756 \\
\hline Adjusted Straw Yield & 4042 & 5064 & 5575 & 5720 \\
\hline Income (Paddy) & 54,306 & 68,387 & 85,523 & 96,453 \\
\hline Income (Straw) & 20,210 & 25,322 & 27,873 & 28,602 \\
\hline Gross Income (Paddy + Straw) & 74,516 & 93,708 & 113,396 & 125,055 \\
\hline Net Benefit (Rs ha' ${ }^{-1}$ ) & 49,891 & 68,603 & 87,811 & $\mathbf{9 8 , 9 9 0}$ \\
\hline
\end{tabular}

DSR = Direct Seeded Rice; CR = Crop Residue; T1 = 0 kg P $\mathrm{O}_{5} \mathrm{ha}^{-1}$; T2 = 40 kg P $\mathrm{O}_{5} \mathrm{ha}^{-1}$; $\mathrm{T} 3=80 \mathrm{~kg} \mathrm{P}_{2} \mathrm{O}_{5} \mathrm{ha}^{-1}$; $\mathrm{T} 4=120 \mathrm{~kg} \mathrm{P}_{2} \mathrm{O}_{5} \mathrm{ha}^{-1} ;+\mathrm{CR}=$ With Crop Residue; - CR = Without Crop Residue. 
Table 3. Partial budget analysis for wheat grown with and without CR under saline soil.

\begin{tabular}{|c|c|c|c|c|}
\hline Average Yield $\left(\mathrm{kg} \cdot \mathrm{ha}^{-1}\right)$ 2012-13 & $\mathbf{T 1}$ & T2 & T3 & T4 \\
\hline \multicolumn{5}{|c|}{$+\mathbf{C R}$} \\
\hline Grains & 2652 & 3194 & 3560 & 3259 \\
\hline Straw & 5836 & 6285 & 6576 & 6358 \\
\hline TCV & 27,125 & 27,605 & 28,085 & 28,565 \\
\hline $10 \%$ less Grain Yield & 265.2 & 319.4 & 356.0 & 325.9 \\
\hline 10\% less Straw Yield & 583.6 & 628.5 & 657.6 & 635.8 \\
\hline Adjusted Grain Yield & 2387 & 2875 & 3204 & 2933 \\
\hline Adjusted Straw Yield & 5252 & 5657 & 5918 & 5722 \\
\hline Income (Grain) & 59,670 & 71,865 & 80,100 & 73,328 \\
\hline Income (Straw) & 42,019 & 45,252 & 47,347 & 45,778 \\
\hline Gross Income (Grain + Straw) & 101,689 & 117,117 & 127,447 & 119,105 \\
\hline Net Benefit (Rs ha' $\left.{ }^{-1}\right)$ & 74,564 & 89,512 & 99,362 & 90,540 \\
\hline \multicolumn{5}{|c|}{$-\mathbf{C R}$} \\
\hline Grains & 1591 & 2054 & 2877 & 3320 \\
\hline Straw & 3577 & 4433 & 5263 & 5832 \\
\hline TVC & 25,125 & 25,605 & 26,085 & 26,565 \\
\hline 10\% less Paddy Yield & 159.1 & 205.4 & 287.7 & 332.0 \\
\hline 10\% less Straw Yield & 357.7 & 443.3 & 526.3 & 583.2 \\
\hline Adjusted Grain Yield & 1432 & 1849 & 2589 & 2988 \\
\hline Adjusted Straw Yield & 3219 & 3990 & 4737 & 5249 \\
\hline Income (Grain) & 35,798 & 46,215 & 64,733 & 74,700 \\
\hline Income (Straw) & 25,754 & 31,918 & 37,894 & 41,990 \\
\hline Gross Income (Grain + Straw) & 61,552 & 78,133 & 102,626 & 116,690 \\
\hline Net Benefit (Rs ha ${ }^{-1}$ ) & 36,427 & 52,528 & 76,541 & 90,125 \\
\hline
\end{tabular}

$+\mathrm{CR}=$ With Crop Residue; $-\mathrm{CR}=$ Without Crop Residue; $\mathrm{T} 1=0 \mathrm{~kg} \mathrm{P}_{2} \mathrm{O}_{5} \mathrm{ha}^{-1} ; \mathrm{T} 2=40 \mathrm{~kg} \mathrm{P}_{2} \mathrm{O}_{5} \mathrm{ha}^{-1}$; $\mathrm{T} 3=80 \mathrm{~kg} \mathrm{P}_{2} \mathrm{O}_{5} \mathrm{ha}^{-1} ; \mathrm{T}_{4}=120 \mathrm{~kg} \mathrm{P}_{2} \mathrm{O}_{5}$ ha $^{-1}$.

\subsection{Cost Benefit Ratio (CBR), Net Benefit (NB) and Marginal Rate of Return (MRR)}

The data in Table 4 and Table 5 indicates that maximum CBR (4.9) for direct seeded rice was calculated with $80 \mathrm{~kg} \mathrm{P}_{2} \mathrm{O}_{5} \mathrm{ha}^{-1}$ under crop residues incorporation and it was 4.8 with higher rate of $\mathrm{P}_{2} \mathrm{O}_{5}\left(120 \mathrm{~kg} \cdot \mathrm{ha}^{-1}\right.$ without crop residues incorporation). Similarly, the highest CBR of 4.5 for wheat was calculated with $80 \mathrm{~kg} \mathrm{P}_{2} \mathrm{O}_{5}$ ha $^{-1}$ under crop residues incorporation and it was 4.4 with higher rate of $\mathrm{P}_{2} \mathrm{O}_{5}\left(120 \mathrm{~kg} \cdot \mathrm{ha}^{-1}\right.$ without crop residues incorporation). Generally, all $\mathrm{P}$ application rates along with crop residues incorporation showed much higher NB and highest residual value being the maximum NB with $80 \mathrm{~kg} \mathrm{P}_{2} \mathrm{O}_{5} \mathrm{ha}^{-1}$ application to direct seeded rice (Rs = 108,680/-) and wheat (Rs = 99,362/-) crops. Among $\mathrm{P}$ application treatments without crop residues incorporation, the maximum NB (Rs $=98,990 /-$ and Rs $=90,125 /-)$ and highest residual values $(72,925$ and 63,560) for direct seeded rice and wheat respectively, were obtained with higher $\mathrm{P}$ application rate $\left(120 \mathrm{~kg} \mathrm{P}_{2} \mathrm{O}_{5} \mathrm{ha}^{-1}\right)$ which were not again as much as that of $80 \mathrm{~kg}_{2} \mathrm{O}_{5} \mathrm{ha}^{-1}$ application with crop residues incorporation. Similarly Table 6 showed that highest MRR (4063) for direct seeded rice was computed with $40 \mathrm{~kg}_{2} \mathrm{O}_{5}$ ha $^{-1}$ under crop residues incorporation and while it was 3950 with $80 \mathrm{~kg} \mathrm{P}_{2} \mathrm{O}_{5} \mathrm{ha}^{-1}$ without crop residues incorporation). Whereas Table 7 indicated, the highest MRR (3114) for wheat was calculated with $40 \mathrm{~kg} \mathrm{P}_{2} \mathrm{O}_{5}$ ha $^{-1}$ under crop residues incorporation and it was (4179) with $80 \mathrm{~kg} \mathrm{P}_{2} \mathrm{O}_{5} \mathrm{ha}^{-1}$ without crop residues incorporation).

On the basis of this investigation, it is concluded that crop residues incorporation is the best choice rather it's 
Table 4. Cost Benefit Ratio (CBR) for direct seeded rice grown under saline soil.

\begin{tabular}{|c|c|c|c|c|}
\hline Treatments & Gross Income (Paddy + Straw) & TCV & NB & CBR \\
\hline \multicolumn{5}{|c|}{$+\mathbf{C R}$} \\
\hline T1 $\left(0 \mathrm{~kg} \mathrm{P}_{2} \mathrm{O}_{5} \mathrm{ha}^{-1}\right)$ & 101,710 & 26,625 & 75,085 & 3.8 \\
\hline T2 $\left(40 \mathrm{~kg} \mathrm{P}_{2} \mathrm{O}_{5} \mathrm{ha}^{-1}\right)$ & 121,694 & 27,105 & 94,589 & 4.5 \\
\hline T3 $\left(80 \mathrm{~kg} \mathrm{P}_{2} \mathrm{O}_{5} \mathrm{ha}^{-1}\right)$ & 136,265 & 27,585 & 108,680 & 4.9 \\
\hline T4 (120 kg P $\left.\mathrm{O}_{5} \mathrm{ha}^{-1}\right)$ & 129,285 & 28,065 & 101,220 & 4.6 \\
\hline \multicolumn{5}{|c|}{$-\mathbf{C R}$} \\
\hline T1 $\left(0 \mathrm{~kg} \mathrm{P}_{2} \mathrm{O}_{5} \mathrm{ha}^{-1}\right)$ & 74,516 & 24,625 & 49,891 & 3.0 \\
\hline T2 (40 kg $\left.\mathrm{P}_{2} \mathrm{O}_{5} \mathrm{ha}^{-1}\right)$ & 93,708 & 25,105 & 68,603 & 3.7 \\
\hline T3 (80 kg $\left.\mathrm{P}_{2} \mathrm{O}_{5} \mathrm{ha}^{-1}\right)$ & 113,396 & 25,585 & 87,811 & 4.4 \\
\hline T4 (120 kg P $\left.\mathrm{O}_{5} \mathrm{ha}^{-1}\right)$ & 125,055 & 26,065 & 98,990 & 4.8 \\
\hline
\end{tabular}

TCV = Total Cost that Vary; NB = Net Benefit; CBR = Cost Benefit Ratio; +CR = With Crop Residue; $-\mathrm{CR}=$ Without Crop Residue.

Table 5. Cost Benefit Ratio (CBR) for wheat grown under saline soil.

\begin{tabular}{ccccc}
\hline Treatments & Gross Income (Grain + Straw) & TCV & NB & CBR \\
\hline T1 $\left(0 \mathrm{~kg} \mathrm{P}_{2} \mathrm{O}_{5} \mathrm{ha}^{-1}\right)$ & +CR & & 3.7 \\
T2 $\left(40 \mathrm{~kg} \mathrm{P}_{2} \mathrm{O}_{5} \mathrm{ha}^{-1}\right)$ & 101,689 & 27,125 & 74,564 & 4.2 \\
T3 $\left(80 \mathrm{~kg} \mathrm{P}_{2} \mathrm{O}_{5} \mathrm{ha}^{-1}\right)$ & 117,117 & 27,605 & 89,512 & 4.5 \\
T4 $\left(120 \mathrm{~kg} \mathrm{P}_{2} \mathrm{O}_{5} \mathrm{ha}^{-1}\right)$ & 127,447 & 28,085 & 99,362 & 4.2 \\
\hline & 119,105 & 28,565 & 90,540 & 2.4 \\
T1 $\left(0 \mathrm{~kg} \mathrm{P}_{2} \mathrm{O}_{5} \mathrm{ha}^{-1}\right)$ & $-\mathbf{C R}$ & & 3.1 \\
T2 $\left(40 \mathrm{~kg} \mathrm{P}_{2} \mathrm{O}_{5} \mathrm{ha}^{-1}\right)$ & & 25,125 & 36,427 & 3.9 \\
T3 $\left(80 \mathrm{~kg} \mathrm{P}_{2} \mathrm{O}_{5} \mathrm{ha}^{-1}\right)$ & 78,133 & 25,605 & 52,528 & 76,541 \\
T4 $\left(120 \mathrm{~kg} \mathrm{P}_{2} \mathrm{O}_{5} \mathrm{ha}^{-1}\right)$ & 102,626 & 26,085 & 90,125 & 4.4 \\
\hline
\end{tabular}

TCV = Total Cost that Vary; NB = Net Benefit; CBR = Cost Benefit Ratio; +CR = With Crop Residue; $-\mathrm{CR}=$ Without Crop Residue.

Table 6. Marginal Rate of Return (MRR) for direct seeded rice grown under saline soil.

\begin{tabular}{|c|c|c|c|c|c|}
\hline Treatments & TCV & MC & NB & MNB & MRR \\
\hline \multicolumn{6}{|c|}{$+\mathbf{C R}$} \\
\hline T1 (0 kg P $\left.\mathrm{P}_{5} \mathrm{ha}^{-1}\right)$ & 26,625 & - & 75,085 & - & - \\
\hline T2 $\left(40 \mathrm{~kg} \mathrm{P}_{2} \mathrm{O}_{5} \mathrm{ha}^{-1}\right)$ & 27,105 & 480 & 94,589 & 19,504 & 4063 \\
\hline T3 $\left(80 \mathrm{~kg} \mathrm{P}_{2} \mathrm{O}_{5} \mathrm{ha}^{-1}\right)$ & 27,585 & 960 & 108,680 & 33,595 & 3499 \\
\hline T4 (120 kg P $\left.\mathrm{O}_{5} \mathrm{ha}^{-1}\right)$ & 28,065 & 1440 & 101,220 & 26,135 & 1815 \\
\hline \multicolumn{6}{|c|}{$-\mathbf{C R}$} \\
\hline T1 (0 kg P $\left.\mathrm{P}_{5} \mathrm{ha}^{-1}\right)$ & 24,625 & - & 49,891 & - & - \\
\hline T2 (40 kg $\left.\mathrm{P}_{2} \mathrm{O}_{5} \mathrm{ha}^{-1}\right)$ & 25,105 & 480 & 68,603 & 18,712 & 3898 \\
\hline T3 (80 kg $\left.\mathrm{P}_{2} \mathrm{O}_{5} \mathrm{ha}^{-1}\right)$ & 25,585 & 960 & 87,811 & 37,920 & 3950 \\
\hline T4 (120 kg P $\left.\mathrm{P}_{5} \mathrm{ha}^{-1}\right)$ & 26,065 & 1440 & 98,990 & 49,099 & 3410 \\
\hline
\end{tabular}

TCV = Total Cost that Vary; MC = Marginal Cost; NB = Net Benefit; MNB = Marginal Net Benefit; MRR = Marginal Rate of Return; +CR = With Crop Residue; $-\mathrm{CR}=$ Without Crop Residue. 
burning to improve direct seeded rice and wheat yields with $80 \mathrm{~kg} \mathrm{P}_{2} \mathrm{O}_{5} \mathrm{ha}^{-1}$ application under slightly saline soil.

\subsection{Residual Analysis for Direct Seeded Rice and Wheat Crops}

Residual analysis is done to verify the results of marginal analysis. The results of residual analysis (Table 8 and Table 9) demonstrate that the highest residual values of direct seeded rice and wheat were observed with (80 $\mathrm{kg}$ $\mathrm{P}_{2} \mathrm{O}_{5}$ ha $^{-1}$ and crop residues incorporation) followed by $120 \mathrm{~kg} \mathrm{P}_{2} \mathrm{O}_{5} \mathrm{ha}^{-1}$ without crop residues incorporation. The improvement in their economics is definitely attributed to continuous $\mathrm{P}$ application and crop residues incorporation that might have altered the soil physical conditions due to which $\mathrm{P}$ availability and its utilization was enhanced. Consequently, the nutrient utilization efficiency positively happened to be a factor for healthy growth and yield of direct seeded rice and wheat crops under adverse soil condition. This could be supported by the findings of [20]-[24], who had documented similar points of view regarding better correlation between nutrients and plant growth under improved soil physical conditions. The similar trend in economic analysis results of mungbean cultivars and $\mathrm{P}$ application rates have been reported by [25]. Performance of green gram and response functions as influenced by different levels of nitrogen and phosphorous was computed by [26]. Similarly the results of [27] showed that application of inorganic fertilizers and organic manures enhanced nutrients availability and improved economical production of mungbean. On the basis of all economic analyses of research work data

Table 7. Marginal Rate of Return (MRR) for wheat grown under saline soil.

\begin{tabular}{|c|c|c|c|c|c|}
\hline Treatments & TCV & MC & NB & MNB & MRR \\
\hline \multicolumn{6}{|c|}{$+\mathbf{C R}$} \\
\hline T1 $\left(0 \mathrm{~kg} \mathrm{P}_{2} \mathrm{O}_{5} \mathrm{ha}^{-1}\right)$ & 27,125 & - & 74,564 & - & - \\
\hline T2 $\left(40 \mathrm{~kg} \mathrm{P}_{2} \mathrm{O}_{5} \mathrm{ha}^{-1}\right)$ & 27,605 & 480 & 89,512 & 14,948 & 3114 \\
\hline T3 $\left(80 \mathrm{~kg} \mathrm{P}_{2} \mathrm{O}_{5} \mathrm{ha}^{-1}\right)$ & 28,085 & 960 & 99,362 & 24,798 & 2583 \\
\hline T4 (120 kg $\left.\mathrm{P}_{2} \mathrm{O}_{5} \mathrm{ha}^{-1}\right)$ & 28,565 & 1440 & 90,540 & 15,976 & 1109 \\
\hline \multicolumn{6}{|c|}{$-\mathbf{C R}$} \\
\hline T1 $\left(0 \mathrm{~kg} \mathrm{P}_{2} \mathrm{O}_{5} \mathrm{ha}^{-1}\right)$ & 25,125 & - & 36,427 & - & - \\
\hline T2 $\left(40 \mathrm{~kg} \mathrm{P}_{2} \mathrm{O}_{5} \mathrm{ha}^{-1}\right)$ & 25,605 & 480 & 52,528 & 16,101 & 3354 \\
\hline T3 $\left(80 \mathrm{~kg} \mathrm{P}_{2} \mathrm{O}_{5} \mathrm{ha}^{-1}\right)$ & 26,085 & 960 & 76,541 & 40,114 & 4179 \\
\hline T4 (120 kg $\left.\mathrm{P}_{2} \mathrm{O}_{5} \mathrm{ha}^{-1}\right)$ & 26,565 & 1440 & 90,125 & 53,698 & 3729 \\
\hline
\end{tabular}

TCV = Total Cost that Vary; MC = Marginal Cost; NB = Net Benefit; MNB = Marginal Net Benefit; MRR = Marginal Rate of Return; +CR = With Crop Residue; - CR = Without Crop Residue.

Table 8. Analysis using Residual for direct seeded rice grown under saline soil.

\begin{tabular}{|c|c|c|c|c|}
\hline Treatments & $1 \mathrm{TCV}$ & $2 \mathrm{NB}$ & $\begin{array}{l}3 \text { Returned Required by } \\
\text { Farmer }(100 \% * 1) \text { Rs ha }^{-1}\end{array}$ & $\begin{array}{c}\quad 4=[2-3] \\
\left.\text { Residual (Rs ha }{ }^{-1}\right)\end{array}$ \\
\hline \multicolumn{5}{|c|}{$+\mathbf{C R}$} \\
\hline T1 (0 kg $\left.\mathrm{P}_{2} \mathrm{O}_{5} \mathrm{ha}^{-1}\right)$ & 26,625 & 75,085 & 26,625 & 48,460 \\
\hline $\mathbf{T} 2\left(40 \mathrm{~kg} \mathrm{P}_{2} \mathrm{O}_{5} \mathrm{ha}^{-1}\right)$ & 27,105 & 94,589 & 27,105 & 67,484 \\
\hline T3 (80 kg $\left.\mathrm{P}_{2} \mathrm{O}_{5} \mathrm{ha}^{-1}\right)$ & 27,585 & 108,680 & 27,585 & 81,095 \\
\hline T4 (120 kg $\left.\mathrm{P}_{2} \mathrm{O}_{5} \mathrm{ha}^{-1}\right)$ & 28,065 & 101,220 & 28,065 & 73,155 \\
\hline \multicolumn{5}{|c|}{$-\mathbf{C R}$} \\
\hline T1 (0 kg $\left.\mathrm{P}_{2} \mathrm{O}_{5} \mathrm{ha}^{-1}\right)$ & 24,625 & 49,891 & 24,625 & 25,266 \\
\hline $\mathbf{T} 2\left(40 \mathrm{~kg} \mathrm{P}_{2} \mathrm{O}_{5} \mathrm{ha}^{-1}\right)$ & 25,105 & 68,603 & 25,105 & 43,498 \\
\hline T3 (80 kg $\mathrm{P}_{2} \mathrm{O}_{5} \mathrm{ha}^{-1}$ ) & 25,585 & 87,811 & 25,585 & 62,226 \\
\hline T4 (120 kg $\left.\mathrm{P}_{2} \mathrm{O}_{5} \mathrm{ha}^{-1}\right)$ & 26,065 & 98,990 & 26,065 & 72,925 \\
\hline
\end{tabular}

TCV = Total Cost that Vary; NB = Net Benefit; +CR = With Crop Residue; - CR = Without Crop Residue. 
Table 9. Analysis using Residual for wheat grown under saline soil.

\begin{tabular}{|c|c|c|c|c|}
\hline Treatments & $1 \mathrm{TCV}$ & 2 NB & $\begin{array}{l}3 \text { Returned Required by Farmer } \\
(100 \% * 1) \text { Rs ha }^{-1}\end{array}$ & $\begin{array}{c}4=[2-3] \\
\text { Residual }\left(\mathrm{Rs} \mathrm{ha}^{-1}\right)\end{array}$ \\
\hline \multicolumn{5}{|c|}{$+\mathbf{C R}$} \\
\hline T1 $\left(0 \mathrm{~kg} \mathrm{P}_{2} \mathrm{O}_{5} \mathrm{ha}^{-1}\right)$ & 27,125 & 74,564 & 27,125 & 47,439 \\
\hline T2 $\left(40 \mathrm{~kg} \mathrm{P}_{2} \mathrm{O}_{5} \mathrm{ha}^{-1}\right)$ & 27,605 & 89,512 & 27,605 & 61,907 \\
\hline T3 $\left(80 \mathrm{~kg} \mathrm{P}_{2} \mathrm{O}_{5} \mathrm{ha}^{-1}\right)$ & 28,085 & 99,362 & 28,085 & 71,277 \\
\hline T4 (120 kg P $\left.\mathrm{P}_{2} \mathrm{O}_{5} \mathrm{ha}^{-1}\right)$ & 28,565 & 90,540 & 28,565 & 61,975 \\
\hline \multicolumn{5}{|c|}{$-\mathbf{C R}$} \\
\hline T1 $\left(0 \mathrm{~kg} \mathrm{P}_{2} \mathrm{O}_{5} \mathrm{ha}^{-1}\right)$ & 25,125 & 36,427 & 25,125 & 11,302 \\
\hline T2 (40 kg $\left.\mathrm{P}_{2} \mathrm{O}_{5} \mathrm{ha}^{-1}\right)$ & 25,605 & 52,528 & 25,605 & 26,923 \\
\hline T3 $\left(80 \mathrm{~kg} \mathrm{P}_{2} \mathrm{O}_{5} \mathrm{ha}^{-1}\right)$ & 26,085 & 76,541 & 26,085 & 50,456 \\
\hline T4 (120 kg P $\left.\mathrm{P}_{5} \mathrm{ha}^{-1}\right)$ & 26,565 & 90,125 & 26,565 & 63,560 \\
\hline
\end{tabular}

TCV = Total Cost that Vary; NB = Net Benefit; +CR = With Crop Residue; - CR = Without Crop Residue.

(2011-2012), the incorporation of crop residues and $80 \mathrm{~kg} \mathrm{P}_{2} \mathrm{O}_{5} \mathrm{ha}^{-1}$ could be recommended to farmers to get maximum return by growing direct seeded rice and wheat on marginally salt-affected soils. The findings could also be supported by the results of [28].

\section{Conclusion}

Our results indicated that $\mathrm{P}$ application @ $80 \mathrm{~kg}_{2} \mathrm{O}_{5} \mathrm{ha}^{-1}$ along with crop residues incorporation (2 ton $\mathrm{ha}^{-1}$ ) was found to be superior to rest of the treatments in term of producing maximum grain yield of both direct seeded rice and wheat crop grown under marginally saline soil.

\section{References}

[1] Ghafoor, A., Qadir, M. and Murtaza, G. (2004) Salt Affected Soils: Principal of Management. Allied Book Centre, Urdu Bazar, Lahore, 304 p.

[2] Abbas, H.A. (2009) General Agriculture. 4th Edition, Urdu Bazar, Lahore, 127 p.

[3] GOP (2014) Pakistan Bureau of Statistics, Economic Affairs Division, Central Statistical Office, Islamabad.

[4] Ali, A., Arshadullah, M., Hyder, S.I., Mahmood, I.A. and Zaman, B. (2012) Rice Productivity and Soil Health as Affected by Wheat Residue Incorporation along with Nitrogen Starter Dose under Salt-Affected Soil. Pakistan Journal of Agricultural Research, 25, 257-265.

[5] Gupta, R.K., Naresh, R.K., Hobbs, P.R., Jiaguo, Z. and Ladha, J.K. (2003) Sustainability of Post Green Revolution Agriculture: The Rice-Wheat Cropping System of the Indo-Gangetic Plains and China. Proceedings of an International Symposium of Improving the Productivity and Sustainability of Rice-Wheat Systems: Issues and Impacts, Charlotte, 22 October 2001, 1-25.

[6] FAO (2012) Fertilizer Prices and Profitability of Fertilizer Use. Chapter 7, 28.

[7] Byous, E.W., Williuams, J.E., Jonesa, G.E., Horwath, W.R. and Kessel, C. (2004) Nutrient Requirements of Rice with Alternative Straw Management. Better Crops, 36, 6-11.

[8] Eagle, A.J., Bird, J.A., Horwaath, W.R., Linquist, B.A., Brouder, S.M., Hill, J.E. and Kessel, C.V. (2000) Rice Yield and Nitrogen Utilization Efficiency under Alternative Straw Management Practices. Agronomy Journal, 92, 1096-1103. http://dx.doi.org/10.2134/agronj2000.9261096x

[9] Mishra, B.N., Sharma, P.K. and Bronson, K.F. (2001) Decomposition of Rice Straw and Mineralization of Carbon, Nitrogen, Phosphorus and Potassium in Wheat Field Soil in Western Uttar Pardesh. Journal of the Indian Society of Soil Science, 49, 419-424.

[10] Mahmood, I.A., Ali, A., Aslam, M., Shahzad, A., Sultan, T. and Hussain, F. (2013) Phosphorus Availability in Different Salt-Affected Soils as Influenced by Crop Residue Incorporation. International Journal of Agriculture and Biology, 15, 472-478.

[11] Anjum, K., Qadir, I., Azhar, M.F. and Hafeez, S. (2013) Economic Evaluation of Irrigated Plantation in Kamalia, Pun- 
jab, Pakistan. Journal of Agricultural Research, 51, 189-202.

[12] Rath, A.K., Ramakrishnan, B., Rao, V.R. and Sethunathan, N. (2005) Effects of Rice Straw and Phosphorus Application on Production and Emission of Methane from Tropical Rice Soil. Journal of Plant Nutrition and Soil Science, 168, 248-254. http://dx.doi.org/10.1002/jpln.200421604

[13] Danga, B.O. and Wakindiki, I.I.C. (2009) Effect of Placement of Straw Mulch on Soil Conservation, Nutrient Accumulation, and Wheat Yield in a Humid Kenyan Highland. Journal of Tropical Agriculture, 47, 30-36.

[14] Sainio, P.P., Kontturi, M. and Peltonen, J. (2006) Phosphorus Seed Coating Enhancement on Early Growth and Yield Components in Oat. Agronomy Journal, 98, 206-211. http://dx.doi.org/10.2134/agronj2005.0141

[15] Arshadullah, M., Ali, A., Hyder, S.I. and Khan, A.M. (2012) Effect of Wheat Residue Incorporation along with N Starter Dose on Rice Yield and Soil Health under Saline Sodic Soil. The Journal of Animal and Plant Sciences, 22, 753-757.

[16] Slaton, N.A., Wilson, C.E., Norman, R.J., Ntamatungiro, S. and Frizzell, D.L. (2002) Rice Response to Phosphorus Fertilizer Application Rate and Timing on Alkaline Soils in Arkansas. Agronomy Journal, 94, 1393-1399. http://dx.doi.org/10.2134/agronj2002.1393

[17] Sharma, S.N. and Prasad, R. (2003) Yield and P Uptake by Rice and Wheat Grain in a Sequence as Influenced by Phosphate Fertilization with Diammonium Phosphate and Mussoorie Rock Phosphate with or without Crop Residue and Phosphate Solubilizing Bacteria. The Journal of Agricultural Science, 141, 359-369. http://dx.doi.org/10.1017/S0021859603003678

[18] Krishna, G.M.A., Misra, A.K.K., Hati, K.M., Bandyopadhyay, K.K., Ghosh, P.K. and Mohanty, M. (2004) Rice Residue Management Options and Effects on Soil Properties and Crop Productivity. Journal of Food, Agriculture and Environment, 2, 224-231.

[19] Aslam, M., Flowers, T.J., Qureshi, R.H. and Yeo, A.R. (2008) Interaction of Phosphate and Salinity on the Growth and Yield of Rice (Oryza sativa L.). Journal of Agronomy and Crop Science, 176, 249-258. http://dx.doi.org/10.1111/j.1439-037X.1996.tb00469.X

[20] Gillani, S.M.W., Ahmad, A.H., Khalid, F., Zamir, M.S.I., Anwar, M.B., Ikram, W. and Jabbar, A. (2014) Impact of Nutrient Management on Growth, Yield and Quality of Forage Maize (Zea mays L.) under Agroclimatic Conditions of Faisalabad. Journal of Agricultural Research, 52, 499-510.

[21] Mehdi, S.M., Sajjad, N., Sarfraj, M., Hassan, B.Y.K.G. and Sadiq, M. (2003) Response of Wheat to Different Phosphatic Fertilizers in Varying Textured Salt Affected Soils. Journal of Applied Sciences, 3, 474-480. http://dx.doi.org/10.3923/jas.2003.474.480

[22] Araujo, A.P. and Teixeira, M.G. (2008) Relationships between Grain Yield and Accumulation of Biomass, Nitrogen and Phosphorus in Common Bean Cultivars. Revista Brasileira de Ciência do Solo, 32, 1977-1986. http://dx.doi.org/10.1590/S0100-06832008000500019

[23] Saha, S., Saha, B., Sidhu, M., Pati1, S. and Roy, P.D. (2014) Grain Yield and Phosphorus Uptake by Wheat as Influenced by Long-Term Phosphorus Fertilization. African Journal of Agricultural Research, 9, 607-612. http://dx.doi.org/10.5897/AJAR2013.7525

[24] Ramzam, A., Noor, T., Khan, T.N. and Hina, A. (2014) Correlation, Cluster and Regression Analysis of Seed Yield and Its Contributing Trait in Pea (Pisum sativum L.). Journal of Agricultural Research, 52, 481-488.

[25] Khan, A.H. (2004) Effect of Cultivar and Phosphorous Rates on Growth, Adiation Interception and Yield of Mungbean (Vigna radiata L.). Master's Thesis, Department of Agronomy, University of Agriculture, Faisalabad.

[26] Srinivas, M. and Shaik, M. (2002) Performance of Green Gram and Response Functions as Influenced by Different Levels of Nitrogen and Phosphorous. Crop Research (Hisar), 24, 458-462.

[27] Naeem, M., Iqbal, J. and Bakhsh, M.A.A. (2006) Comparative Study of Inorganic Fertilizers and Organic Manures on Yield and Yield Components of Mungbean (Vigna radiat L.). Journal of Agriculture \& Social Sciences, 2, 227-229.

[28] Ayyaz, F., Anjum, K., Qadir, I., Nouman, W., Afzal, S. and Asif, M. (2014) Best Economic Rotation of Farm Trees in Tehsil Muzzaffargarh. Journal of Agricultural Research, 52, 569-579. 